\title{
Dynamic Self-organization in an Open Reaction Network as a Fundamental Mechanism for the Emergence of Life
}

\author{
Yoshiharu Mukouyama ${ }^{1}$ and Yoshihiro Nakato*2 \\ ${ }^{1}$ Division of Science, College of Science and Engineering, Tokyo Denki University, \\ Hatoyama, Saitama 350-0394, Japan \\ mukoyama@mail.dendai.ac.jp \\ ${ }^{2}$ Department of Materials Engineering Science, Graduate School of Engineering Science, \\ Osaka University, Toyonaka, Osaka 560-8531, Japan (presently retired) \\ nakato@chem.es.osaka-u.ac.jp
}

\begin{abstract}
The emergence of life on the earth has attracted intense attention but the mechanism of it still remains an unsolved question. A key problem is that it has been left unclear why a living organism, which is regarded as an open reaction system, can demonstrate dynamic self-organization leading to highly-ordered structures and adaptive and evolutionary behavior. This paper shows by computer simulation that an open reaction network, which is characterized as a network of flexible constituent elements and irreversible processes, is converted to a self-organized system with adaptive and evolutionary ability when it has reached a fully-balanced stationary state. Strikingly, this result indicates that dynamic self-organization spontaneously emerges in a prebiotic chemical system placed under constant thermodynamic forces according to the second law of thermodynamics, not against it. The dynamic self-organization has potential for producing highly ordered chemical structures through evolution and is expected to have played a fundamental role in the emergence of life on the primitive earth.
\end{abstract}

\section{Introduction}

The emergence of life on the earth is one of the most important topics in recent scientific research. A great number of studies has been made and a lot of interesting findings has been reported about this issue. ${ }^{1-6}$ However, no sufficiently convincing explanation has still been given to the mechanism of it. ${ }^{4}$

A key problem is that it has been left unclear why a living organism, which is regarded as an open reaction system, can have autonomous self-organizing ability leading to the production of highly-ordered structures and the emergence of adaptive and evolutionary behavior. Some of scientists say that the emergence of life on the earth happened by lucky accidents under favorable conditions ${ }^{7}$ but such an idea is too optimistic. It cannot give a meaningful probability of the emergence of life because it needs repeated occurrence of lucky accidents until the first living organism emerges. Moreover, the idea can never explain the emergence of free independent spirit in living organisms because a reaction system studied under this idea is in an entirely passive state against an attack of the external world and has no ability to organize itself.

Thus, it is indispensable to clarify how dynamically self-organizing ability emerged 
in a prebiotic chemical system. In fact, many approaches have been proposed for this purpose, such as general systems theory, ${ }^{8}$ the autopoiesis system, ${ }^{9}$ hypercycle, ${ }^{10}$ synergetics, ${ }^{11}$ dissipative structures, ${ }^{12,13}$ and complexity science. ${ }^{5,14-16}$ For example, a large number of studies have been made on dissipative structures but phenomena studied till now are still fairly far in quality and character from self-organization in living organisms. A diversity of theoretical models has also been constructed in complexity science such as logistic equation, cellular automaton, and network theory. However, the adaptive and evolutionary behavior of complex systems in complex science arises from algorithm in theoretical models and it remains unclear how such algorithm can be explained by physicochemical laws in real substances.

The present work investigates the behavior of an open reaction network by computer simulation. The aim is to clarify whether it can demonstrate dynamic self-organization with adaptive and evolutionary ability. As an open reaction network is regarded as a fundamental structure of living organisms, this issue should be of critical importance. However, almost no attention has been paid to it. This is most probably because there has been intense belief that the emergence of dynamic self-organization needs a special mechanism for overcoming disturbances by the second law of thermodynamics such as synergetics, ${ }^{11}$ autocatalysis,${ }^{12,13}$ the edge of chaos, ${ }^{14-16}$ etc. Certainly, it appears that a simple open reaction network has no such special mechanism. However, an open reaction network can be converted to a self-organized system with adaptive and evolutionary ability when it has reached a stationary state and therefore has potential for producing highly ordered chemical structures through evolution. This finding has opened a possibility that an open reaction network played a fundamental role in the emergence of the primitive life on the earth.

\section{Results}

Recent studies of the origin of life have disclosed a promising possibility that the first living organism emerged in a small inorganic compartment in the vicinity of hydrothermal vents in a primitive deep sea. ${ }^{17,18}$ If various chemical substances, discharged from a hydrothermal vent and produced by following reactions in a deep sea, diffused into such a small inorganic compartment, they will have formed an open reaction network there. Figure 1 shows a model of such an open reaction network in a small inorganic compartment, used for computer simulation. Circles stand for chemical substances, different numerals referring to different chemical sub- stances. A pair of arrows shows a chemical reaction. Now, a chemical substance indicated by a numeral $i$ in Figure 1 is hereafter described as $\mathrm{R}_{i}$. Thus, Figure 1 shows that $R_{1}$ and $R_{2}$ react and

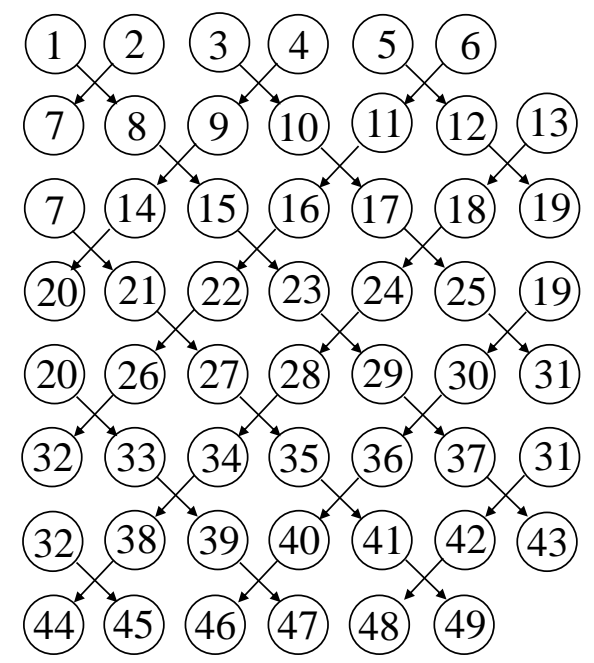

Figure 1. A simulation model of an open reaction network. 
produce $R_{7}$ and $R_{8}$, and so on. All reactions in Figure 1 are assumed to be elementary reactions and thus their rate $\mathcal{R}_{\mathrm{r}}$ can be expressed by second-order rate equations. For example, the rate of a reaction of $R_{1}$ and $R_{2}$, producing $R_{7}$ and $R_{8}$, is given as follows

$$
\mathcal{R}_{\mathrm{r}}=k_{\mathrm{f}} C_{1} C_{2}-k_{\mathrm{b}} C_{7} C_{8}
$$

where $C_{i}$ refers to the concentration of $\mathrm{R}_{i}$ and $k_{\mathrm{f}}$ and $k_{\mathrm{b}}$ are rate constants for the forward and the backward processes of reaction, respectively. On the other hand, the rate of inflow or outflow, $\mathcal{R}_{\mathrm{d} i}$, for a chemical substance $\mathrm{R}_{i}$ can be approximately expressed, based on Fick's law of diffusion, as follows.

$$
\mathcal{R}_{\mathrm{d} i}=k_{\mathrm{d} i}\left(C_{i}^{\mathrm{ex}}-C_{i}^{\mathrm{in}}\right)
$$

where $k_{\mathrm{d} i}$ is the rate constant for the inflow or outflow of $\mathrm{R}_{i}$, and $C_{i}^{\mathrm{ex}}$ and $C_{i}^{\text {in }}$ are the concentrations of $\mathrm{R}_{i}$ in the exterior and the interior (i.e. an open reaction network), respectively. (The superscript "in" for $C_{i}^{\text {in }}$ is omitted in case no confusion happens.) The diffusion of organic molecules in an aqueous solution is in general much faster than their reactions in the absence of effective catalysts and thus it can be assumed that organic molecules in an open reaction network have a uniform spatial distribution.

Figure 2 shows time courses of the concentrations of chemical substances, obtained by using an ODE (= ordinary differential equation) solver in MATLAB software (MathWorks). Calculation conditions are as follows: the external concentrations (concentrations in the exterior) of chemical substances entering an open reaction network $\left(R_{1}\right.$ to $R_{6}$ and $\left.R_{13}\right)$ range between 2 and $8 \times 10^{-3} \mathrm{M}\left(\mathrm{M}=\mathrm{mol} \mathrm{dm}{ }^{-3}\right)$, while those of outgoing reaction products $\left(R_{43}\right.$ to $\left.R_{49}\right)$ are $5 \times 10^{-4} M$ for all. These concentrations are kept constant and therefore an open reaction network is placed under a constant thermodynamic force, as explained later. The initial concentrations of chemical substances in an open reaction network are chosen to be $1 \times 10^{-3} \mathrm{M}$ for all. The rate constants $k_{\mathrm{f}}$ for reactions vary between 0.6 and $1.1 \mathrm{M}^{-1} \mathrm{~s}^{-1}$, with $k_{\mathrm{f}} / k_{\mathrm{b}}$ ranging from 1.4 to 2.6 , while the rate constants $k_{\mathrm{d} i}$ for inflows or outflows of chemical substances are chosen between 0.94 and $1.0 \times 10^{-3} \mathrm{~s}^{-1}$.

We can see from Figure 2 that the concentrations of chemical substances in an open reaction network initially change in a complex way but gradually approach constant and finally be- come constant, indicating that an open reaction net- work reaches a stationary state. Figure 3 shows a concentration profile of chemical substances in a stationary state, obtained at a time of $t$ $=5 \times 10^{5} \mathrm{~s}$.

Calculations were done under various conditions. A stationary state was

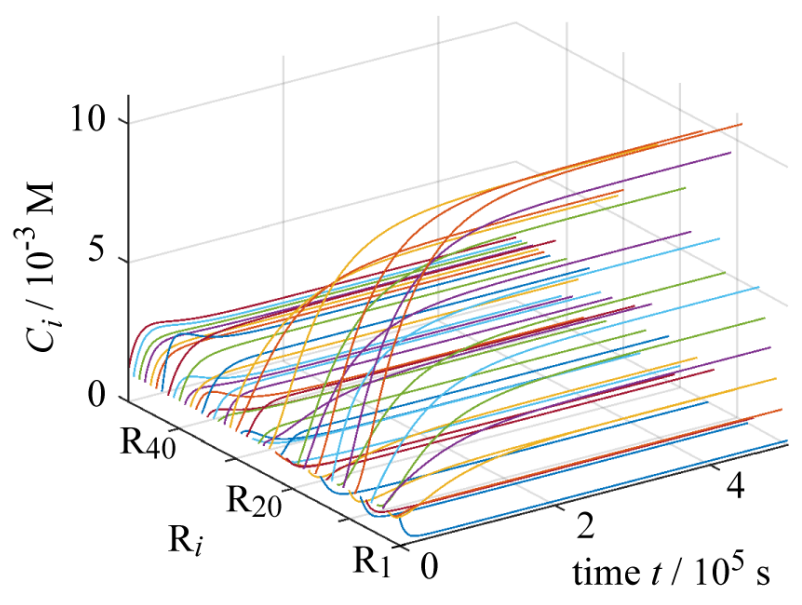

Figure 2. Time courses of the concentrations of chemical substances in an open reaction network of Figure 1. 
achieved in all cases thus far investigated, including a case in which all chemical substances $\left(R_{1}\right.$ to $R_{49}$ ) can diffuse out of a reaction network. A concentration profile of chemical substances in a stationary state little depended on parallel increases or decreases of the external concentrations of source substances but largely depended on changes in their relative concentrations. Similarly, a concentration profile in a stationary state little depended on parallel increases or decreases in $k_{\mathrm{f}}$ and $k_{\mathrm{d} i}$ but largely depended on changes in their relative values. Interestingly, a concentration profile in a stationary state was kept unchanged even when the initial concentrations of chemical substances in an open reaction network were decreased to zero, indicating that an open reaction network of Figure 1 has ability to reproduce itself.

To investigate how an open reaction network reaches a stationary state and what properties a stationary state has, we calculated the rate of entropy production $\sigma$ by using the following equations. ${ }^{13}$

$$
\begin{aligned}
& \sigma=\sigma_{1}+\sigma_{2} \\
& \sigma_{1}=\sum_{i} k_{\mathrm{d} i} V\left(C_{i}^{\mathrm{ex}}-C_{i}^{\mathrm{in}}\right)\left(\mu_{i}^{\mathrm{ex}}-\mu_{i}^{\mathrm{in}}\right) / T \\
& \sigma_{2}=\sum_{j} v_{j}\left(A_{j} / T\right)
\end{aligned}
$$

where $\sigma_{1}$ refers to the rate of entropy production by inflows and outflows of chemical substances, while $\sigma_{2}$ does that by chemical reactions in an open reaction network. In equation ( $3 \mathrm{a}), \mu_{i}^{\mathrm{ex}}$ and $\mu_{i}^{\mathrm{in}}$ are chemical potentials of $\mathrm{R}_{i}$ in the exterior and the interior (an open reaction network), respectively. $V$ is the volume of an open reaction network system and $T$ the temperature. On the other hand, $v_{j}$ in equation ( $\left.3 \mathrm{~b}\right)$ refers to the rate of the $j$-th reaction, defined by

$$
v_{j}=d \xi_{j} / d t=d\left(n_{j p} / v_{j p}\right) / d t
$$

where $\xi_{j}$ is the degree of extent of the $j$-th reaction, $\mathrm{d} n_{j p}$ a change in the amount of substance for a chemical substance $\mathrm{R}_{p}$ by the $j$-th reaction, and $v_{j p}$ the stoichiometric coefficient for $\mathrm{R}_{p}$ in the $j$-th reaction ( $v_{j p}$ is defined as negative for reactants and positive for products). According to this definition, $\mathcal{R}_{\mathrm{r}}$ in equation (1) is expressed as $v / V$. The quantity $A_{j}$ in equation (3b) is the affinity of the $j$-th reaction, ${ }^{13}$ given as $A_{j}=-\sum_{p} v_{j p} \mu_{p}$. For example, the affinity $A$ of a reaction of $\mathrm{R}_{1}$ and $\mathrm{R}_{2}$, producing $\mathrm{R}_{7}$ and $\mathrm{R}_{8}$, is given as

$$
A=\left(\mu_{1}+\mu_{2}\right)-\left(\mu_{7}+\mu_{8}\right)=R T \ln \left(k_{\mathrm{f}} C_{1} C_{2} / k_{\mathrm{b}} C_{7} C_{8}\right)
$$

where $R$ is the gas constant. The absolute value of the affinity, $|A|$, decreases as a 
reaction approaches equilibrium, and $A=0$ at equilibrium. The reaction rate $v_{j}$ can be expressed by using $A_{j}$ as follows ${ }^{13}$

$$
v_{j}=v_{j f}\left\{1-\exp \left(-A_{j} / R T\right)\right\}
$$

where $v_{j \mathrm{f}}$ is the rate of the forward process of the $j$-th reaction. Equation (3e) indicates that $v_{j}$ is in proportion to $\left(A_{j} / T\right)$ when $\left|A_{j} / R T\right| \ll 1$, where $\left(A_{j} / T\right)$ is the thermodynamic force for the $j$-th reaction. Thus, we can say that the $j$-th reaction is in a nearequilibrium linear region when $\left|A_{j} / R T\right| \ll 1 .^{13}$ The thermodynamic force for a whole open reaction network $X_{\mathrm{RN}}$ is given by

$$
X_{\mathrm{RN}}=\left(\sum_{i} A_{\mathrm{d} i}+\sum_{j} A_{j}\right) / T
$$

where $A_{\mathrm{d} i}=\left|\mu_{i}^{\mathrm{ex}}-\mu_{i}^{\mathrm{in}}\right|$ is the affinity for the inflow or outflow of $\mathrm{R}_{i}$. A simple consideration shows that the quantity $\left(\sum_{i} A_{\mathrm{d} i}+\sum_{j} A_{j}\right)$ for an open reaction network is determined by the chemical potentials (or the concentrations) of entering and outgoing chemical substances in the exterior and is constant under the condition of Figure 2. Thus, an open reaction network of Figure 2 is placed under a constant thermodynamic force, as mentioned earlier.

Figure 4 shows time courses of entropy-production rates, $\sigma_{1}, \sigma_{2}$ and $\sigma$, in an open reaction network of Figure 1 at 293.15K, obtained simultaneously with those of concentrations of chemical substances shown in Figure 2. $\sigma_{1}$ initially increases and then decreases and becomes constant, while $\sigma_{2}$ starts from a high value and decreases and then increases and becomes constant. Thus, the sum of them, $\sigma=\sigma_{1}+\sigma_{2}$, monotonously decreases with time and becomes constant. This result can be explained as follows. Under the calculation conditions of Figure 2, the reaction rates of chemical substances in an open reaction network, $\mathcal{R}_{\mathrm{r}}$, are slightly higher than the rates of their inflows, $\mathcal{R}_{\mathrm{d}}$, at $t=0$. Therefore, chemical substances in an open reaction network first start to react with one another, and hence the $\sigma_{2}$ value starts from a high value and decreases by a decrease in their concentrations, as mentioned above. Then, inflows of chemical substances happen, accompanied by an increase in the $\sigma_{1}$ value. Such inflow soon reaches a peak and decreases because the concentrations of entering chemical substances in an open reaction network increase and come close to those in the exterior. Thus, the $\sigma_{1}$ value also reaches a peak and decreases. Finally, resultant increases in the concentrations of entering chemical substances in an open reaction network lead to an increase in the $\sigma_{2}$ value because of increases in reacting chemical substances in an open reaction network.

In general, the $\sigma_{1}$, $\sigma_{2}$ and $\sigma$ values change in a rather complex man-

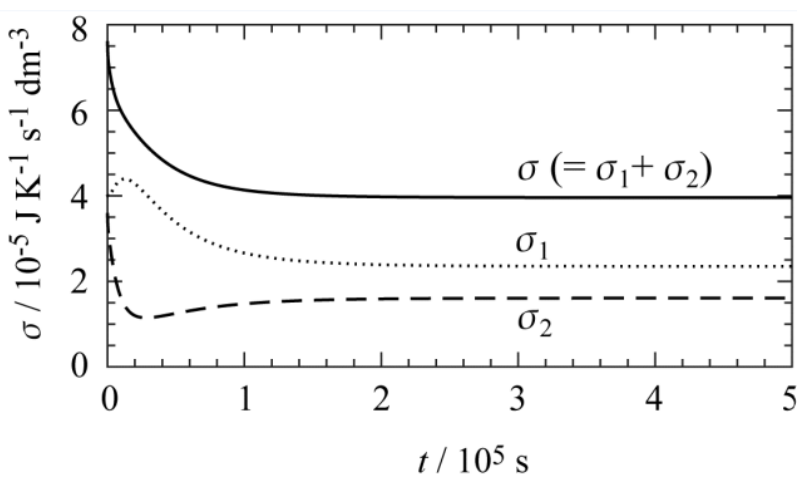

Figure 4. Time courses of the entropy-production rates, $\sigma_{1}, \sigma_{2}$ and $\sigma$, in an open reaction network of Figure 1 at $293.15 \mathrm{~K}$, obtained simultaneously with those of concentrations of chemical substances shown in Figure 2. 
ner, especially in the initial stage, because they are strongly affected by increases and decreases in entering and outgoing chemical substances and reacting ones, as men- tioned above. Thus, these values often increase along a convex curve just before they finally become constant, as seen in the $\sigma_{2}$ value of Figure 4. An important point is that they become constant in every case after the lapse of a sufficiently long time. The attainment of constant $\sigma_{1}, \sigma_{2}$ and $\sigma$ means the achievement of constant reaction and diffusion rates, again indicating that an open reaction network finally reaches a stationary state.

We investigated the behavior of open reaction networks with different structures from Figure 1. All systems thus far investigated, including systems of a small size and those in which all reactions are catalyzed by chemical substances in it, showed similar behavior to an open reaction network of Figure 1, indicating that results of Figure 2 to 4 are typical of the behavior of an open reaction network.

\section{Discussion}

The time evolution of an open reaction network is described by a set of nonlinear differential equations, each consisting of a combination of equations (1) and (2). A problem is that such a set of differential equations is in general complex and actually impossible to solve analytically. Thus, in the present work we investigated the behavior of an open reaction network by computer simulation. As displayed in Figure 2, computer simulation clearly shows that an open reaction network such as Figure 1 spontaneously changes and finally reaches a stationary state, at which the concentrations $C_{i}$ of chemical substances are all kept constant everywhere

$$
d C_{i} / d t=0 \text {. }
$$

The same result was obtained for an open reaction network in which all chemical substances can diffuse out of it and for one in which all reactions are catalyzed by chemical substances in it, as mentioned earlier. Accordingly, we can say that open reaction networks of various types reach a stationary state.

Why can an open reaction network reach a stationary state? This problem can be explained qualitatively based on equations (1) and (2) as follows. The concentration of a chemical substance at a high-concentration site in a reaction network continues to decrease because such a site is in general surrounded with a negative concentration gradient in the outward direction. On the other hand, the concentration of a chemical substance at a low-concentration site continues to increase because such a site is in general surrounded with a negative concentration gradient. Such a decrease and an increase continue until they are balanced with one another.

The attainment of a stationary state in an open reaction network can also be seen from the achievement of constant entropy production rates $\left(\sigma_{1}, \sigma_{2}\right.$ and $\left.\sigma\right)$ in large $t$, as shown in Figure 4. In relation to this result, there is an important point to be noted. As mentioned earlier, the $\sigma_{1}, \sigma_{2}$ and $\sigma$ values indeed finally become constant but often increase just before they become constant, as is exemplified in the $\sigma_{2}$ value of Figure 4. Computer simulation shows that such behavior is observed, irrespective of whether an open reaction network is in a near-equilibrium linear region or not. On the other hand, it is well known that Prigogine reported that the entropy production rate $\sigma$ in a closed or open system placed under constant thermodynamic forces, lying in a near-equilibrium linear region, spontaneously decreases and takes a minimum at a stationary state. ${ }^{13}$ Thus, here is a clear discrepancy.

This can be explained as follows. At first, an increase in the entropy-production rate 
$\sigma_{2}$ for a chemical reaction can be explained in the following. For a chemical reaction described as $\mathrm{R}_{1}+\mathrm{R}_{2} \rightarrow \mathrm{P}_{1}+\mathrm{P}_{2}$, the entropy-production rate $v(A / T)$ can be expressed from equation ( $3 \mathrm{~b}$ ) as follows,

$$
\sigma_{2}=R V\left(k_{\mathrm{f}} C_{\mathrm{r} 1} C_{\mathrm{r} 2}-k_{\mathrm{b}} C_{\mathrm{p} 1} C_{\mathrm{p} 2}\right) \ln \left(k_{\mathrm{f}} C_{\mathrm{r} 1} C_{\mathrm{r} 2} / k_{\mathrm{b}} C_{\mathrm{p} 1} C_{\mathrm{p} 2}\right)
$$

where $C_{\mathrm{r} 1}$ and $C_{\mathrm{r} 2}$ are concentrations of reactants $\mathrm{R}_{1}$ and $\mathrm{R}_{2}$ while $C_{\mathrm{p} 1}$ and $C_{\mathrm{p} 2}$ are those of products $P_{1}$ and $P_{2}$. Thus, the progress of a chemical reaction leads to decreases in $C_{\mathrm{r} 1}$ and $C_{\mathrm{r} 2}$ and increases in $C_{\mathrm{p} 1}$ and $C_{\mathrm{p} 2}$ and hence leads to a decrease in the $\sigma_{2}$ value for this reaction. However, the progress of a chemical reaction leads to increases in the concentrations of reactants of following reactions and decreases in the concentrations of products of preceding reactions, and thus leads to increases in the $\sigma_{2}$ values for these reactions. Inflows of chemical substances from the exterior also lead to increases in in the $\sigma_{2}$ values. Accordingly, if the latter contribution exceeds the former in an open reaction network, the $\sigma_{2}$ value increases. This happens even in a final stage at which the $\sigma_{2}$ value just becomes constant. A similar argument applies to an increase in the $\sigma_{1}$ value. Nevertheless, the above argument is not necessarily in contradiction to Prigogine's conclusion. This is because the above argument also indicates that reaction and diffusion processes always happen in the direction of approaching equilibrium and hence they have approached equilibrium to the largest extent after a stationary state (or a full balance) has been attained. This means that the entropy production rate takes a minimum in a stationary state, in agreement with Prigogine's conclusion. Note here that this conclusion never means that we can say that an open reaction network changes so that the entropy production rate decreases and reaches a stationary state.

The fact that an open reaction network reaches a stationary state indicates that a stationary state is stable. The above conclusion that the entropy production rate takes a minimum in a stationary state also means that a stationary state is stable. The stability of a stationary state of an open reaction network can be further confirmed by the Lyapunov method. ${ }^{13}$ Let us consider a function $L=\sum_{i} \delta C_{i}{ }^{2}, \delta C_{i}=C_{i}-C_{i}^{\mathrm{s}}$, where $C_{i}$ and $C_{i}^{\mathrm{s}}$ are the concentration of a chemical substance $\mathrm{R}_{i}$ in a fluctuated state and in a stationary state, respectively. It is obvious that $L$ is positive.

$$
L=\sum_{i} \delta C_{i}^{2}>0
$$

In addition, rate equation (1) indicates that when $C_{i}$ becomes higher than $C_{i}^{\mathrm{s}}$ by a fluctuation, the rate of a decomposition reaction of $\mathrm{R}_{i}$ increases, namely when $C_{i}-$ $C_{i}^{\mathrm{s}}>0, d C_{i} / d t<0$ because $d C_{i} / d t=0$ in a stationary state. Similarly, when $C_{i}$ becomes lower than $C_{i}^{\mathrm{s}}$ by a fluctuation, the rate of a decomposition reaction of $\mathrm{R}_{i}$ decreases, namely when $C_{i}-C_{i}^{\mathrm{s}}<0, d C_{i} / d t>0$. The same argument holds for diffusional motion, which follows rate equation (2). Thus, we obtain

$$
d L / d t=\sum_{i} 2\left(C_{i}-C_{i}^{\mathrm{s}}\right) d C_{i} / d t<0
$$

Equations (6a) and (6b) indicate that the function $L$ meets the conditions of Lyapunov stability, i.e. a stationary state of an open reaction network is stable.

Now, it has become clear that an open reaction network spontaneously reaches a stationary state and a stationary state is stable against a fluctuation. This conclusion has various important meanings. Firstly, this conclusion means that a stationary state has a self. A stationary state incessantly interacts with the surroundings and chemical substances in it always change but nevertheless a stationary state keeps a full balance between inner processes unchanged. This means that a fully-balanced state acts as a self of a stationary state. 
Secondly, the above conclusion that a stationary state is stable against a fluctuation means that it has ability to organize itself. Namely, when a stationary state fluctuates by an attack of the surroundings, such a destabilized stationary state can return to the original stationary state. A stationary state is stabilized by a network of irreversible chemical processes and realizes a harmonious state with the surroundings.

Thirdly, if a stationary state has ability to organize itself, the attainment of a stationary state means the production of a new organized thing. In general, reaction and diffusion processes in a chemical system only follow individual laws about reactions and diffusion and happen rather independently. On the other hand, reaction and diffusion processes in a stationary state not only follow such individual laws but also behave so that they maintain a fully-balanced state as a whole. Otherwise, a stationary state could not be stable. This means that chemical substances in a stationary state have acquired new ability to stabilize a stationary state as a whole. The attainment of a stationary state can thus be regarded as emergence. There is a jump between a non-stationary state and a stationary one. Interestingly, in contrast to chemical substances in a stationary state, constituent elements of a machine such as a computer and a humanoid robot in general only follow individual rules and has no ability to stabilize a machine as a whole.

There is another important point to be noted. If a destabilized stationary state has ability to organize itself, as mentioned above, this means that a stationary state has ability to adapt itself to a new environment and evolve. The self-organizing ability of an open reaction network leads to adaptive and evolutionary behavior.

In conclusion, the foregoing arguments have revealed that an open reaction network, even a simple one consisting of non-catalyzed secondary reactions, spontaneously reaches a stationary state and comes to have self-organizing ability. The self-organizing ability leads to adaptive and evolutionary behavior, similar to that of living organisms, ${ }^{19}$ and has large potential for producing a diversity of ordered structures. Moreover, an open reaction network investigated in the present work is easily formed in a prebiotic chemical system under common conditions and is expected to have played a fundamental role in the emergence of life on the primitive earth.

\section{References}

1. Meierhenrich, U. J., Filippi, J. -J., Meinert, C., Vierling, P. \& Dworkin, J. P. On the origin of primitive cells: from nutrient intake to elongation of encapsulated nucleotides. Angew. Chem. Int. Ed. 49, 3738-3750 (2010).

2. Adamala, K. \& Szostak, J. W. Competition between model protocells driven by an encapsulated catalyst. Nature Chemistry 5, 495-501 (2013).

3. Perunov, N., Marsland, R. A. \& England, J. L. Statistical physics of adaptation. Phys. Rev. X 6, 021036 (2016).

4. Luisi, P. L. The Emergence of Life -from Chemical Origins to Synthetic Biology 2nd ed. (Cambridge Univ. Press, Cambridge, 2016).

5. Smith, E. \& Morowitz, H. J. The Origin and Nature of Life on Earth: The Emergence of the Fourth Geosphere (Cambridge Univ. Press, Cambridge, 2016).

6. Deamer, D. W. Assembling Life: How Can Life Begin on Earth and Other Habitable Planets? (Oxford Univ. Press, Oxford, 2019).

7. Monod, J. L. Le hasard et la nécessité, Essai sur la philosophie naturelle de la biologie modern (Alfred A. Knopf, Inc, Paris, 1971). 
8. von Bertalanffy, L. General system theory: foundations, development, applications (George Braziller, New York, 1968).

9. Varela, F. J., Maturana, H. R. \& Uribe, R. B. Autopoiesis: the organization of living system, its characterization and a model. Biosystems 5, 187-196 (1974).

10. Eigen, M. \& Schuster, P. The Hypercycle: A Principle of Natural Self-Organization (Springer, Berlin, 1979).

11. Haken, H. The science of structure: synergetics (Van Nostrand Reinhold, New York, 1984).

12. Prigogine, I. \& Nicolis, G. Self-organization in non-equilibrium systems (Wiley, New York, 1977).

13. Prigogine, I. \& Kondepudi, D. Thermodynamique - des moteurs thermiques aux structures dissipatives (Odile Jacob, Paris, 1999).

14. Kauffman, S. At home in the universe: The search for laws of self-organization and complexity (Oxford University Press, Oxford, 1995).

15. Levin, R. Complexity Life at the edge of chaos 2nd ed. (The University of Chicago Press, Chicago, 1999).

16. Mitchell, M. Complexity: A guided tour (Oxford University Press, Oxford, 2009).

17. Koonin, E. V. \& Martin, W. On the origin of genomes and cells within inorganic compartments. Treads in Genetics 21, 647-654 (2005).

18. Baaske, P., Weinert, F. M., Duhr, S., Lemke, K. H., Russell, M. J. \& Braun, D. Extreme accumulation of nucleotides in simulated hydrothermal pore systems. Proc. Natl. Acad. Sci. USA 104, 9346-9351 (2007).

19. Alberts, B., Johnson, A., Lewis, J., Morgan, D., Raff, M., Roberts, K., Walter, P., Wilson, J. \& Hunt, T. Molecular biology of the cell 6th ed. (Garland Science, New York, 2014). 